\title{
MÉTODOS DE ANÁLISE DO MOVIMENTO: APLICAÇÕES NA AÇÃO PIANÍSTICA
}

\author{
Daniel da Silva ${ }^{2}$ \\ Maria Bernardete Castelan Póvoas ${ }^{3}$
}

Palavras-chave: ação pianística; coordenação motora; feedback; método de análise.

Resumo: Através de um estudo que trata dos processos de fornecimento feedback durante a aprendizagem motora e uma pesquisa dos principais métodos científicos da área biomecânica, esta investigação traz argumentos interdisciplinares que buscam compreender e otimizar a prática instrumental. Foram descritas técnicas de medição como a cinemetria, eletromiografia e antropometria, com foco na aplicação em procedimentos experimentais do movimento, considerando-se seu emprego na avaliação do trabalho músico-instrumental.

\section{Introdução}

Atualmente, nota-se uma crescente tendência na realização de pesquisas interdisciplinares que trazem o diálogo entre diversas áreas do conhecimento e o campo artístico. Muitas destas pesquisas tratam do desenvolvimento e análise do trabalho, com resultados obtidos através de métodos de mensuração e protocolos de análise definidos.

Métodos de investigação do movimento humano, em especial dos métodos de análise característicos da Biomecânica, quando aplicados no contexto da prática instrumental abrem possibilidades de uma melhor compreensão da estrutura de realização do movimento, assim como de uma aprendizagem mais efetiva e fundamentada. Essa compreensão do movimento, que é direcionada pelos objetivos de cada investigação, inclui conhecimentos sobre os movimentos utilizados em uma determinada ação, movimentos estes que podem envolver a combinação de diferentes segmentos do corpo. Sendo assim, para se atingir determinada meta por meio da utilização do movimento mais eficiente é necessário que se tenha informações acerca da sua estrutura. Este fato mostra que a interdisciplinaridade no trabalho científico deve permite uma melhor compreensão das ações.

No contexto da prática instrumental, a compreensão dos aspectos envolvidos na produção do movimento mostra-se essencial na organização de estratégias de estudo mais eficien-

\footnotetext{
${ }^{1}$ Vinculado ao Projeto de Pesquisa CEART-UDESC: Ação Pianística e Coordenação Motora: Relações Interdisciplinares.

${ }^{2}$ Orientadora, Professora do Departamento de Música - Centro de Artes - Florianópolis - SC.

${ }^{3}$ Acadêmico do Curso de Bacharelado em Piano - CEART/UDESC, bolsista de iniciação científica do PROBIC/UDESC.
} 
tes, incluindo o conhecimento dos aspectos anatômicos e fisiológicos do corpo humano. "Estas ciências [como a Anatomia e a Fisiologia] deveriam ser os pilares de sustentação do processo de ensino-aprendizagem dos instrumentos musicais". (KAPLAN, 1987, p.13). Os movimentos participantes na ação instrumental encontram-se interligados em complexas estruturas anatômicas, sendo que somente através de um completo domínio dessas estruturas pode-se atingir um alto nível de eficiência. "A execução instrumental requer habilidades altamente refinadas e um alto grau de conscientização corporal, para que se possa atingir uma realização ótima”. (CIARLINI e RAFAEL, 1994, p.32).

Para que uma habilidade seja executada com sucesso, é necessário que o indivíduo receba informações acerca de seu progresso nessa dada habilidade. A aquisição e a retenção das habilidades motoras ocorrem através do feedback, que são informações sensoriais fornecidas ao indivíduo durante ou após a execução de uma ação. (SCHMIDT e LEE, 2005).

Os métodos de análise (ou medidas de processo) têm como foco principal o aperfeiçoamento de uma habilidade motora, através da observação, descrição e análise dos movimentos executados por indivíduos. É essencial que o pesquisador ou o grupo de pesquisa esteja informado a respeito da anatomia humana e princípios mecânicos que regem os membros e segmentos mais ativos na ação pianística, assim como acerca das habilidades motoras e padrões de movimento. Devido ao alto grau de variabilidade que a reprodução do movimento humano pode apresentar, a análise de um movimento em sua complexidade estrutural deve ser caracterizada por grande objetividade e confiabilidade. (AMADIO et al., 2002). Em se tratando da ação pianística, é importante também conhecer não apenas a proposta do movimento, mas também sobre os fatores de desempenho (RASCH, 1991) inerentes a execução habilidosa deste movimento, tais como, força, fadiga, rapidez do movimento, flexibilidade, entre outros.

\section{Discussão}

Para que uma habilidade seja executada com sucesso é necessário que o indivíduo receba informações acerca de seu progresso nessa dada habilidade. A aquisição e a retenção das habilidades motoras ocorrem através do feedback, que são informações sensoriais fornecidas ao indivíduo durante ou após a execução de uma ação.

Quando a ação planejada não alcança sua meta, esta pode estar sendo prejudicada por algum erro no planejamento do movimento. Neste caso o feedback tem a função de detectar 
quais aspectos estão influenciando nesse desempenho. O feedback intrínseco é fornecido por meio dos órgãos sensoriais (como a audição e a visão) do próprio sujeito que realizou o movimento. De acordo com o nível de habilidade do executante, este tipo de feedback pode fornecer um grande número de informações sobre o movimento realizado, tais como níveis de precisão, postura do corpo e dos membros envolvidos na ação, entre outras; auxilia o músico durante a execução de uma peça, informando na detecção e correção de erros cometidos após ou durante o movimento.

O feedback intrínseco torna-se mais efetivo em ações que envolvem movimentos lentos e voluntários, pois o executante pode fazer compensações durante a ação. Em movimentos rápidos isto não acontece: o tempo de resposta não acompanha o tempo de execução do movimento. (PIEKARZIEVCZ, 2004). Ressalta-se aqui que os movimentos devam ser pensados anteriormente à ação, tornando-se "evidente a necessidade de planejar o movimento antes da ação em função de resultados sonoros previamente estabelecidos, adequando-se movimentos corporais à resolução de aspectos musicais". (PÓVOAS, 1999, p.45). O feedback intrínseco tem, portanto, um papel importante no processo de avaliação do movimento, trazendo à investigação informações relevantes acerca do desempenho. $\mathrm{O}$ executante que possui uma percepção apurada de seus movimentos pode assinalar possíveis erros técnicos ou perceptivos que ocorreram durante a execução, erros que não podem ser apontados em uma simples observação visual.

Se o pianista, aluno ou profissional conceber uma ação corporal inadequada ao início do processo de construção de uma realização instrumental, o padrão automatizado será memorizado e, posteriormente, toda e qualquer correção exigirá tempo e empenho, por vezes maiores do que aqueles anteriormente despendidos, para substituir o padrão. A retenção na memória de padrões reflexos, sejam eles voluntários ou não, se dá por processos análogos. Normalmente, no estágio inicial do estudo do piano, os indivíduos não são capazes de detectar as características relevantes do movimento executado nem a origem de seus erros, embora tenham consciência de que não dominam com destreza tais habilidades.

O feedback extrínseco (ou aumentado) é fornecido ao indivíduo que executou o movimento e informa-lhe a respeito de suas ações; traz informações suplementares e reforça 0 feedback intrínseco sendo, por esta razão, fundamental. Pode ser exercido pelo professor orientador ou por uma gravação áudio-visual e posterior análise do desempenho pianístico que pode ser acompanhada ou não. Esse tipo de feedback dá ao executante informações importantes acerca de seu progresso, estimulando e motivando para que continue sua prática com maior segurança com relação ao possível sucesso de seu desempenho. 
É aconselhável que na transmissão do feedback extrínseco verbal sejam enumeradas prioridades nas informações, pois uma grande quantidade de informações poderá deixar o aluno confuso acerca do que é mais importante se ater naquele momento. Inicialmente, aconselha-se focar a atenção do aprendiz nos padrões mais fundamentais do movimento, direcionando a atenção dele para um ou dois aspectos de cada vez, pois, se o aluno aprender uma ação inadequada à prática ao início do seu processo de estudo, a fixação poderá se tornar tão forte que será difícil esquecer posteriormente o padrão automatizado. Nesse contexto, o feedback intrínseco ainda não é suficiente para que o aluno tome as direções e regulações corretas inerentes do processo de coordenação motora. (MAGILl, 2000). Para esses casos, é fundamental o papel do feedback extrínseco, que traz informações suplementares e reforça o feedback intrínseco.

Consciente da importância do entendimento das diferentes características do movimento, o professor pode selecionar e enfatizar primeiramente o padrão de movimento mais importante envolvido na ação e, depois que o aluno tenha dominado esse padrão, progressivamente fornecer feedback sobre outros aspectos do movimento. (SCHMIDT \& WRISBERG, 2001). No caso da prática pianística, desde o início do estudo de uma peça ou de parte dela, deve-se estar bem informado sobre os passos do processo para aquisição de uma dada habilidade motora e que este processo pode ocorrer de maneira lenta e gradativa, o que requer atenção permanente. Uma forma de se evitar que a tarefa de torne monótona e repetitiva é variar o tipo de estudo, assim como evitar a permanência ininterrupta durante um longo período de tempo em um mesmo trecho de uma peça.

O feedback quando dado após a execução de uma ação correta, é chamado de feedback de reforço. Este tem função de assegurar uma maior confiança na ação desempenhada e aumentar a probabilidade que esta ação será repetida em outra situação semelhante. Thorndike (1927) apud Schmidt e Wrisberg (2001, p. 277) diz que:

\footnotetext{
Uma ação disparada por um estímulo e seguida por conseqüências prazerosas ou recompensadoras tende a se repetir quando o estímulo surgir novamente; uma ação que é seguida por consequiências não prazerosas ou punitivas tende a não ser repetidas.
}

Esse reforço positivo pode ser transmitido tanto de forma verbal, por exemplo: "você está realizando muito bem aqueles stacattos de pulso", como de forma não verbal, por exemplo: "uma expressão facial de aprovação após o término de um trecho difícil". A comunicação 
torna-se mais efetiva quando a mensagem é transmitida envolvendo elementos verbais em conjunto com elementos visuais, valendo também para a mensagem com finalidade corretiva.

A mensagem punitiva, que pode ocorrer durante a instrução de um movimento a fim de notificar o que o aluno fez de errado, pode apresentar-se redundante quando traz a mesma informação do feedback intrínseco, por exemplo, o professor dizendo ao aluno: "você não está tocando bem esse trecho" ou "você errou várias notas durante aquela passagem". Esse conhecimento de resultado, além de não trazer informações relevantes, pode ser desmotivador e até mesmo irritante a alguns alunos. Como estratégia para estimular o aluno, o professor pode perguntar o que ele achou de seu desempenho e, em seguida, apontar os erros indicando formas de corrigi-los, através de dicas e demonstrações de como executar o movimento.

Em conjunto com o feedback extrínseco, deve-se buscar soluções técnicointerpretativas, desenvolvendo assim habilidades para planejar a execução músicoinstrumental. Chiviacowsky e Tani (1997) atentam para a importância de se desenvolver o chamado reforço subjetivo, onde o executante desenvolve a sensibilidade de detecção e correção de seus próprios erros, fornecendo subsídios para que continue mantendo seus níveis de resultado quanto às tarefas desempenhadas. É aconselhável que desde cedo se procure desenvolver a consciência corporal (tensão-relaxamento), através de exercícios de respiração e alongamento, assim como de flexibilidade dos segmentos mais envolvidos na execução pianística: braços, mãos e dedos. Também o feedback auditivo tem um importante papel no processo de memorização, pois permite desenvolver a acuidade relacionada aos parâmetros musicais e influi diretamente no desenvolvimento da leitura e compreensão musical e no desempenho global das peças estudadas. (FINNEY \& PALMER, 2003).

O feedback feito através da gravação áudio-visual é muito recorrente na atividade pianística. Através desse recurso, o músico pode ver seus padrões de movimento com um maior detalhamento, analisando sua prática visualmente e sonoramente. Esse recurso pode ser usado durante as sessões de prática, a fim de avaliar seu desenvolvimento no processo de estudo, assim como para registrar suas apresentações em público, levando em consideração outras variáveis como a acústica do local.

Dentre os métodos de medição e avaliação do movimento utilizados na biomecânica estão: a cinemetria, a eletromiografia e antropometria. Utilizando-se destes métodos, o movimento pode ser descrito e modelado matematicamente, permitindo uma melhor compreensão de sua estrutura.

Através da cinemetria (ou cinematografia) são feitas medições dos movimentos realizados pelo(s) executante(s) através de imagens, registro de trajetórias, decurso de tempo, 
determinação de curvas de velocidade e de aceleração, entre outras variáveis. Para a captação das imagens, os biomecânicos comumente utilizam sistemas de videografia de alta velocidade, articulando as câmeras em diferentes pontos espaciais, a fim de documentar a cinemática do movimento. Com o auxílio de pontos marcados nos eixos articulares, o gesto pode ser digitalizado e reconstruído em duas ou três dimensões. (ALLARD et al., 1995). Lu e O’Connor (1999) destacam que para uma boa avaliação cinemática, é determinante a definição de protocolos experimentais para a colocação dos marcadores. Estes marcadores devem ser posicionados de maneira adequada à estrutura anatômica, eixos articulares, de modo a orientar cada segmento corporal no espaço tridimencional (3D), determinando as variações angulares em cada articulação e respeitando todos os graus de liberdade existentes.

Os sistemas áudio-visuais têm evoluído cada vez mais, podendo-se atualmente, encontrar sistemas de vídeo digitais de alta resolução e alta frequiência a um custo acessível. Entretanto, para uma análise qualitativa que não envolva movimentos muito rápidos, a utilização de uma câmera de vídeo convencional pode atender satisfatoriamente os propósitos da avaliação. Já a análise quantitativa, realizada através da digitalização dos centros articulares do indivíduo, compreende cálculos de grandezas cinéticas e variáveis cinemáticas, o que requer uma instrumentação técnica mais elaborada. (HALL, 1993).

Amadio e Baumann (1990, p.752) apontam alguns dos principais objetivos do procedimento cinemático: “(1) avaliação da técnica para competição, (2) desenvolvimento de técnicas de treinamento, (3) monitoramento de atletas e (4) detecção de talentos esportivos". Considerando-se que o movimento pode apresentar uma grande variabilidade de trajetórias, os indicadores cinemáticos tornam-se, cada vez mais, de grande importância para a avaliação de fatores como a velocidade dos segmentos e articulações, podendo-se determinar as variações da aceleração do movimento, suas variações angulares, assim como outras variáveis concernentes a meta do movimento. (AMADIO et al., 2002).

Outro elemento relevante a ser considerado em qualquer análise biomecânica é o número de tentativas ao se executar um movimento, visto que o grau de proficiência do executante fará com que a cinemática do movimento varie para mais ou para menos, inversamente. É recomendável que sejam feitas outras avaliações depois do experimentopiloto, a fim de ratificar os resultados.

Através da cinética, campo que estuda o movimento dos corpos e suas forças associadas, pode-se analisar a quantidade de força produzida pelos músculos, quantificando assim qual a força apropriada ou necessária a uma ação específica do movimento. Nesse mesmo tipo de análise podem ser considerados fatores antropométricos como o peso e as 
dimensões dos segmentos corporais, quando são utilizados equipamentos como balanças, fita métrica e paquímetros digitais para a obtenção das medidas corporais do indivíduo por meio. Essas medidas são utilizadas para outros métodos de análise, como a cinematografia, por exemplo, e são necessárias para a normatização dos dados e personalização dos modelos físico-matemáticos. (AMADIO et al., 2002). Cada indivíduo possui uma configuração corporal diferente do outro que deve ser levada em consideração no processo de aprendizagem de uma habilidade motora.

A eletromiografia (EMG) é um método utilizado para medição de potenciais elétricos de um músculo ou grupo de músculos, através da verificação dos níveis de participação de cada músculo durante a realização do movimento. Através da eletromiografia é possível identificar-se os músculos que estão sob tensão durante o movimento ou até em estado de "repouso", permitindo assim mapear possíveis fixações musculares desnecessárias ao movimento através de eletrodos que são colocados sobre a pele (discos) ou diretamente no músculo (agulhas).

\section{Conclusões}

A complexidade do movimento humano, em especial da ação pianística, desperta o interesse na investigação sobre o que realmente ocorre no sistema músculo-esquelético. $\mathrm{O}$ grande número de variáveis durante um movimento, em função das características específicas de cada segmento corporal e, sobretudo, de cada indivíduo, determina que uma análise de movimentos estruturalmente tão complexos seja objetiva e efetiva. Através da análise e interpretação do movimento o instrumentista pode apurar seus padrões de movimento e conseqüentemente, seu desempenho artístico. A aquisição e a retenção das habilidades motoras ocorrem através do feedback e o papel do feedback extrínseco é fundamental na prática pianística pois que traz informações suplementares e reforça o feedback intrínseco.

Atualmente encontram-se disponíveis várias ferramentas para o aprimoramento do movimento humano e consciência das estruturas corporais envolvidas na ação. As possibilidades de procedimentos para a análise do movimento estão se tornando mais acessíveis. Os métodos de análise e medição biomecânicos permitem uma maior compreensão dos mecanismos internos reguladores e executores do movimento através de sua descrição, interpretação e análise. A cinemática leva em consideração fatores como velocidade dos segmentos, trajetórias dos eixos articulares, ações dos membros envolvidos na ação e suas 
participações para o sucesso do gesto, entre outros aspectos. A eletromiografia pode ser utilizada para verificar níveis de fadiga e níveis de tensão muscular quando da execução de um movimento.

Vale ressaltar a necessidade de que profissionais da área da música se interessem, cada vez mais, pela cooperação interdisciplinar, em especial com as áreas de Anatomia e a Fisiologia, observando-se a relevância da fundamentação científica, esta embasada em explicações experimentais do movimento, no aprendizado musical.

$\mathrm{Na}$ atividade instrumental, especialmente na área pianística, são poucas as pesquisas biomecânicas cientificamente aplicadas na análise de características do movimento, faltando suporte à formação de teorias com explicação experimental. Visto que nenhuma disciplina se desenvolve por si mesma, este estudo interdisciplinar, em conexão com a atividade instrumental, mostrou novas possibilidades de investigação, constatando-se a necessidade de que mais estudos nesse sentido sejam realizados, buscando-se compreender e otimizar cada vez mais a prática instrumental.

\section{Referências bibliográficas:}

ALLARD, P.; STOKES, A. F.; BLANCHI, J. P. Three-dimensional analysis of human movement. Champaign: Human Kinetics, 1995.

AMADIO,. et al. Métodos de medição em biomecânica do esporte: descrição de protocolos para aplicação no centros de excelência esportiva. Revista Brasileira de Biomecânica: v.3, n.4, p. 57-67, 2002.

AMADIO, Alberto C.; BAUMANN, W. Kinetic and Electromyographical analysis of the triple jump. Sport und Buch Strauss: p. 751-752, 1990.

CIARLINI, Myrian; RAFAEL, Maurílio. O Piano. Campina Grande: LIAA, 1994.

CHIVIACOWSKY, Suzete; TANI, Go. Efeitos da freqüência de conhecimento de resultados na aprendizagem de diferentes programas motores generalizados. Revista Paulista de Educação Física: v.1, n.11, p. 15-26, 1997.

FINNEY, Steven A. ; PALMER, Caroline. Auditory feedback and memory for music performance: Sound evidence for an encoding effect. Memory \& Cognition: v.1, n.31, p. 51-64, 2003.

LU, T-W ; O`CONNOR, J. J. Bone estimation from skin Marker coordinates using global optimation with joint constraints. J. Biomechanics: v.32, p. 129-34, 1999.

HALL, Susan. Biomecânica Básica. Traduzido por Giuseppe Taranto. Rio de Janeiro: Guanabara Koogan, 2005. 
KAPLAN, José A. Teoria da Aprendizagem Pianística. Porto Alegre: Movimento, 1987.

MAGGIL, Richard A. Aprendizagem Motora: conceitos e aplicações. Tradução de Aracy Mendes da Costa. São Paulo: Edgard Blücher, 2000.

PIEKARZIEVCZ, Luiz E. Efeitos do feedback extrínseco aumentado no processo de aprendizagem de uma habilidade motora fechada. Dissertação de Mestrado, 2004, Universidade Federal do Paraná.

PÓVOAS, Maria B. C. Princípio da Relação e Regulação do Impulso-Movimento. Possíveis Reflexos na Ação Pianística. 236 p. Tese de Doutorado, Mímeo, 1999, Universidade Federal do Rio Grande do Sul.

RASCH, Philip J. Cinesiologia e Anatomia Aplicada. Tradução de Marcio Moacyr de Vasconcelos. Rio de Janeiro: Guanabara Koogan, 1991.

SCHMIDT, Richard A.; WRISBERG, Craig A. Aprendizagem e performance motora: uma abordagem de aprendizagem baseada no problema. Tradução de Ricardo Petersen... [et al]. 2th ed. Porto Alegre: Artmed Editora, 2001.

SCHMIDT, Richard A.; LEE, Tim. Motor Control and Learning: a behavioral emphasis. Illinois: Human Kinetics Publishers, 2005. Fourth Edition. 